\title{
$\begin{array}{ll}\text { Preprints are preliminary reports that have not undergone peer review. } \\ \text { Research Square } & \text { They should not be considered conclusive, used to inform clinical practice, }\end{array}$ \\ or referenced by the media as validated information. \\ Performance Investigation of Cooperative Green Underground Wireless Systems
}

\section{R Kanthavel}

King Khalid University

R Dhaya ( $\square$ dhayavel2005@gmail.com )

King Khalid University https://orcid.org/0000-0002-3599-7272

\section{Research Article}

Keywords: Binary phase-shift keying, decode and forward relaying, selection combining, Rayleigh fading, symbol error probability, Green Systems.

Posted Date: September 24th, 2021

DOI: https://doi.org/10.21203/rs.3.rs-911023/v1

License: (c) (1) This work is licensed under a Creative Commons Attribution 4.0 International License. Read Full License 


\section{PERFORMANCE INVESTIGATION OF COOPERATIVE GREEN UNDERGROUND WIRELESS SYSTEMS}

R Kanthavel, Department of Computer Engineering, College of Computer Science, King Khalid University, Abha, Saudi Arabia kanthavel2005@gmail.com

R Dhaya , Department of Computer Science, College of Arts and Science-Sarat Abidha, King Khalid University, Abha, Saudi Arabia dhayavel2005@gmail.com

ABSTRACT: Diversity is a well-known strategy for preventing the negative consequences of fading in Underground wireless communication systems. Space diversity is one of numerous diversity approaches that uses multiple antennas for transmitting and receiving.. Accomplishing spatial diversity in a versatile unit calls for the utilization of different antennas, which, thusly, builds its equipment multifaceted nature and size. Appropriated spatial diversity: versatile units which are circulated in various topographical areas produce numerous correspondence ways. Down to earth execution of dispersed spatial variety necessitates a type of participation among the portable units. A cooperative diversity approach with a sources, multiple transfer, and a purpose is considered in this research. Due to the failure of traditional choice of combination algorithms to consider for the impact of source-to-relay connections in the error analysis of cooperative multiplicity networks, their effectiveness is suboptimal. We have proposed in this research a novel plan which receives another determination system at the goal subsequently giving ideal execution. The start to finish image blunder likelihood (SEP) of a proposed choice technique plot for such a framework, with unravel and forward transferring and parallel stage move entering in a level Rayleigh blurring condition, is broke down. This platform's effectiveness has been investigated using a methodology. This technique was evaluated to a non-cooperative selection combined approach and a standard selection combined strategy. The suggested methodology gives a large sign to- 
commotion proportion improvement over traditional decision joining and noncooperation, according the results.

Keywords: Binary phase-shift keying, decode and forward relaying, selection combining, Rayleigh fading, symbol error probability, Green Systems.

\section{INTRODUCTION}

Remote communication is by far the fastest growing segment of the data and correspondence sector. It has piqued the interest of the media and the public public's unique talent in a business-like manner [1] . The Indian media transmission industry is one of the world's quickest developing businesses with 929.37 million versatile supporters as of May 2012, and furthermore it is the second biggest telecom organize on the planet as far as number of remote associations after China. The expected growth of 5G users from 35 million in 2020 to about 272 million by 2025 is due to demand from 5G users to upgrade their services [2] . As per a survey by Samsung Network, mobile data traffic in India increased by 92 per cent to around 25 petabytes in 2020, of which majority of the traffic is contributed by $4 \mathrm{G} / 5 \mathrm{G}$ users which is a clear indication of ever growing demand for high speed data access.With huge development in voice and information interchanges everywhere throughout the world, the Information and Communication Technology (ICT) industry represents about $2 \%$ or 860 million tons of the world's Green House Gas (GHG) emanations, expanding commitment to the general vitality utilization of the world. This accumulation of GHG, mainly $\mathrm{CO}_{2}$ in the atmosphere leads to the rise in temperature, which is attributed to the effects of global warming. Customary cataclysms like storms, floods and changes in ocean levels are likewise credited to the $\mathrm{CO}_{2}$ fuelled Green House (GH) impact.Every year, over 120,000 new base stations (aka cell phone towers) are deployed, which also increases ambient Electro Magnetic (EM) radiation [3] . So EM radiations can also be considered as pollution to other users and there is an urge to reduce this EM radiation in order to reduce the exposure of human to radiation. Issues mentioned above have made the 'greening' of 
communication an imperative. Having considered low EM radiation and energy efficiency of mobile devices as a means of going green, spectrum is an additional crucial component usage of which requires to be optimized. Arrival of high end mobile devices associate rich apps led to the demand for high speed mobile internet [4] . With growing mobile internet population, there is a constant need to maintain a minimum QoS (in the sense of internet speed) which can be met only if we either increase the number of base stations to cater the high density mobile internet users or to increase the overall bandwidth allocated to each base station [5]. Former solution has direct implications of increased energy consumption and EM radiation; whereas the latter is not always possible as spectral bandwidth is a limited and shared natural resource.

Motivation: Consider an isolated island in sea, where a group of marine biologists who lost track of their path during a marine life explore. Each individual has a personal Underground wireless handset. Now they need to communicate to the base station for their rescue. If the Underground wireless channel between the handset and the base station degrades due to multipath fading, then their communication is lost. To overcome such problems and to enhance the reliability of communication between the Underground wireless handset and the base station, the concept of cooperative diversity can be applied to achieve certain kind of collaboration between the Underground wireless devices. This interesting application of cooperative communication is motivation for this paper.

\section{COOPERATIVE DIVERSITY}

Green correspondence can be accomplished by three methods, as it is appeared in the Figure.1. In urban portable situation where we need high information rate and dependable correspondence, as a general rule, no view way between base station and versatile unit is accessible and furthermore, because of different reflections from mess of encompassing structures, signals from portable unit and base station blur at the separate goals [6]. To counter this issue and give both high information rate and solid 
correspondence with no much vitality and foundation overhead (to make strides toward environmental friendliness), we think about uplink and downlink among versatile and base station as two situations. In uplink scenario, shown in Figure. 2, assuming asymmetric data rate (usually lesser in practice) with downlink, we need reliable communication with base station [7]. We achieve this goal with one or more independent transmission paths between source mobile unit and base station with cooperating relays (other mobile units in vicinity of source mobile unit) retransmitting the overheard data from source mobile to base station [8] .

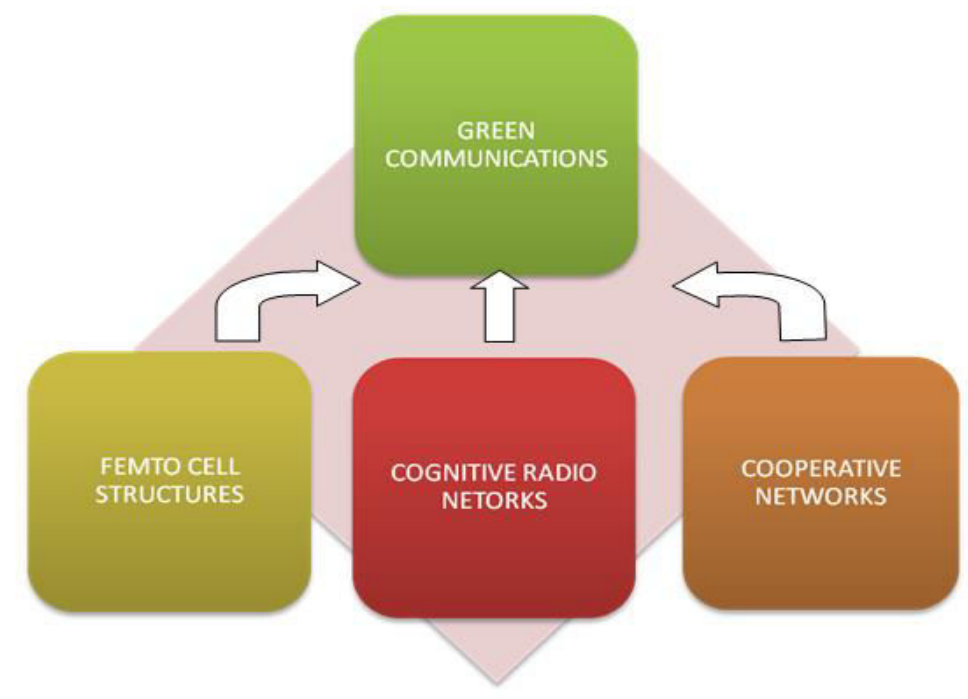

Figure.1. Green communication scenario

If base station could not detect the signal received directly from source mobile because of fading, it could detect the same from one of the retransmissions from relays at later orthogonal (named so, as this retransmission doesn't interfere with other direct transmission) time slots [9]. The implementation of this distributed spatial diversity (as same copy of information is transmitted over diverse space paths) on to the mobile communication system requires some cooperation among the mobile units, relays and base station. Such cooperation in mobile communications is called cooperative diversity [10] . So the first part of this paper objective is, to focus on achieving 'Green' reliable communications through cooperative relay structures by comparing the performance 
analysis of entire cooperative relay structures with the conventionally existing techniques. The cooperative relay ssystem is shown in Figure 2.

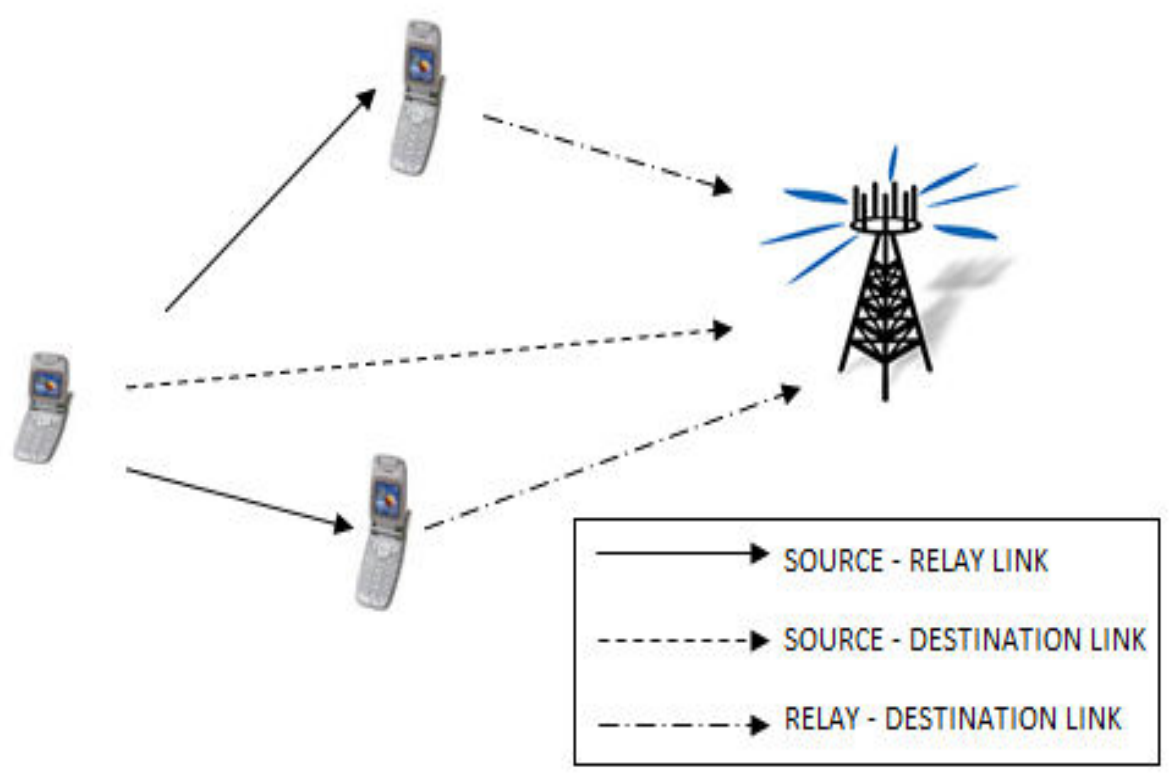

Figure. 2 :Cooperative Relay Structure (Uplink)

Next, in the downlink scenario, where the user usually acts as sink of high data rate information stream from base station, we need both link reliability and data rate[30] . As in uplink scenario, we tackle the problem of unreliable link with co-operating relays [11]. For high data rate, without needing more bandwidth, we introduce Spatial Modulation (SM) at the base station which uses both position of antenna and transmitted symbol from that antenna as a means of information to mobile unit. This is shown in Figure. 3.

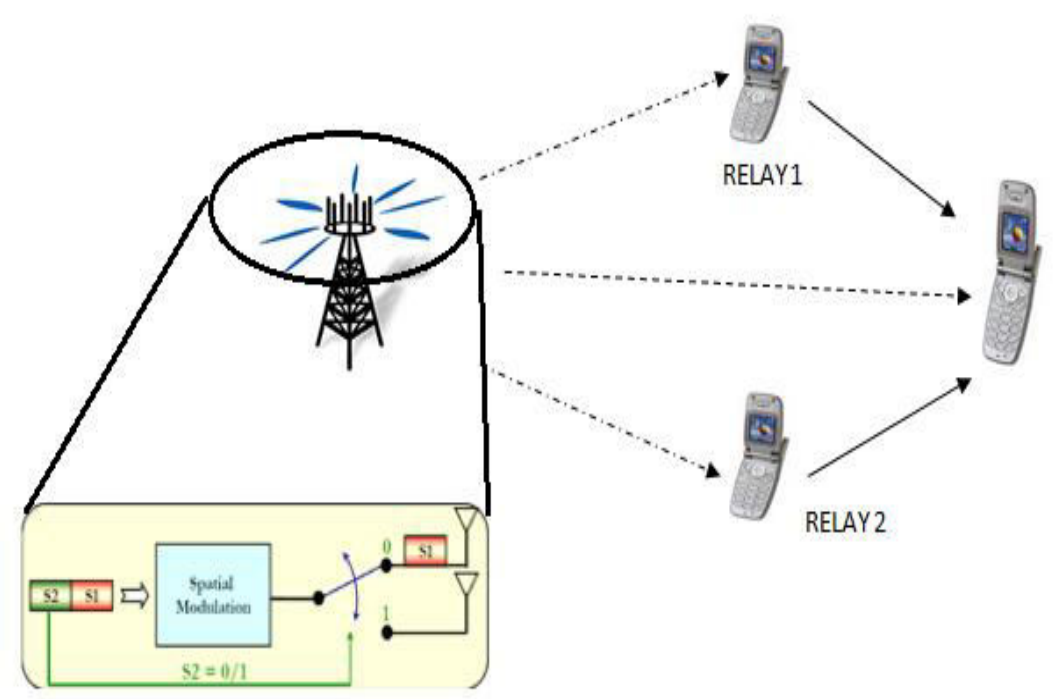


Figure.3 :Cooperative Relay Structure with Spatial Modulation at Base Station

\section{(Downlink)}

The objectives of this paper is to focus on achieving 'Green' reliable communications using relay structures by comparing the performance of entire cooperative relay structures with the conventionally existing techniques; And to design a cooperative Underground wireless network by creating a virtual MIMO systems and analyze its error performance in based on the probability of a signal inaccuracy which improves the recital of a Green Underground wireless systems

\section{EXISTING PROBLEM}

The modeling approach for a single path broadcast, as shown in Figure 4, is described in this existing problem. The modulator, channel, and demodulator blocks are all discussed in this work.

Existing problem in single link transmission: In correspondences industry, remote correspondence is the quickest developing section by any measure. The Indian media transmission industry with 929.37 million portable supporters as of May 2018, is the second biggest telecom arrange on the planet.

Signal Modulation and Modeling: The information being sent is a randomized bipolar bit pattern influenced by either Binary Phase Shift Keying (BPSK) or Quadrature Phase Shift Keying (QPSK) . QPSK, as evidenced by Figure 5, is comprised of two separate (orthogonal) BPSK channels, and so has twice the bandwidth of BPSK [12].

Modelling of a Channel: The information sent from a source to a destination has to travel through to the airspace in an underground wireless network. Numerous factors will corrupt the signals during transmission, as shown in Figure 4. Noise is progressive, while path loss and fading are exponential[29]. 


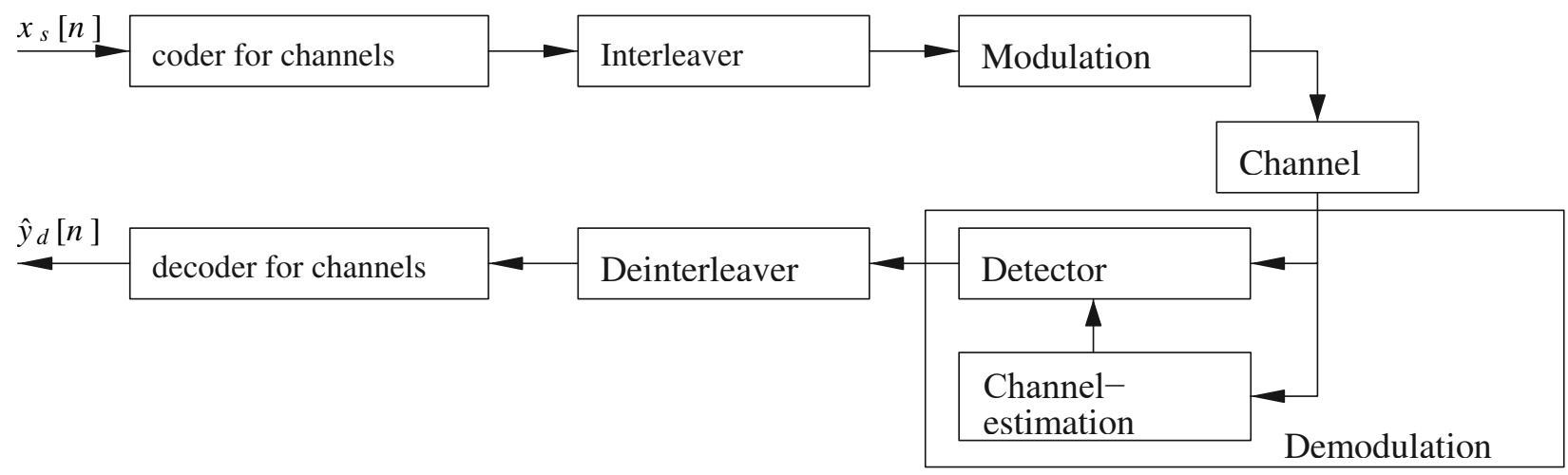

Figure 4: Channel and demodulation block

$y_{d}[n]=h_{s, d}[n] x_{s}[n]+n_{s, d}[n]=p l_{s, d} f_{s, d}[n] x_{s}[n]+n_{s, d}[n]$
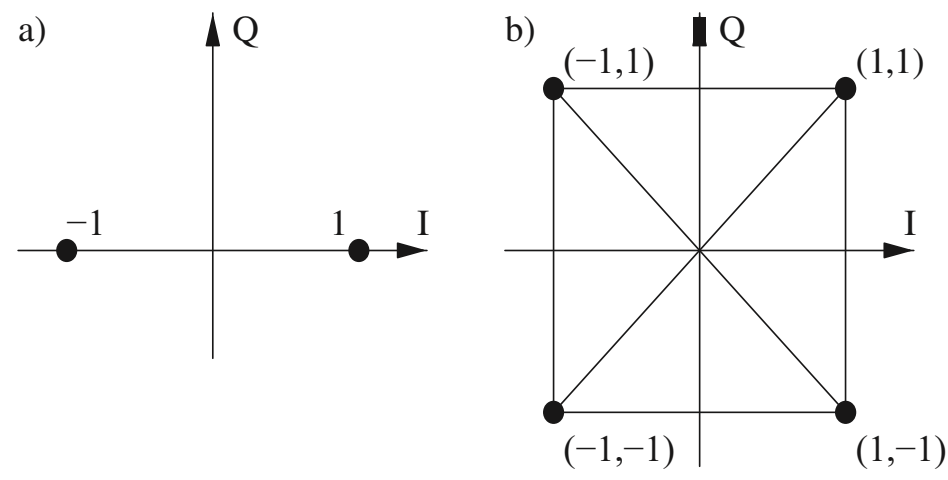

Figure.5 : a) BPSK, b) QPSK,

In Figure 6 shows the design of the channel: path loss $p l_{s, d}$, fading $f s, d[n]$ and noise $n_{s, d}[n] ., s, d$ represent the sender to the intended recipient, The sent sign is $\mathrm{y}_{s}[\mathrm{n}]$, while the received symbol is $\mathrm{x}_{\mathrm{d}}[\mathrm{n}]$.

$y_{s}[n] y_{s, d}[n]$

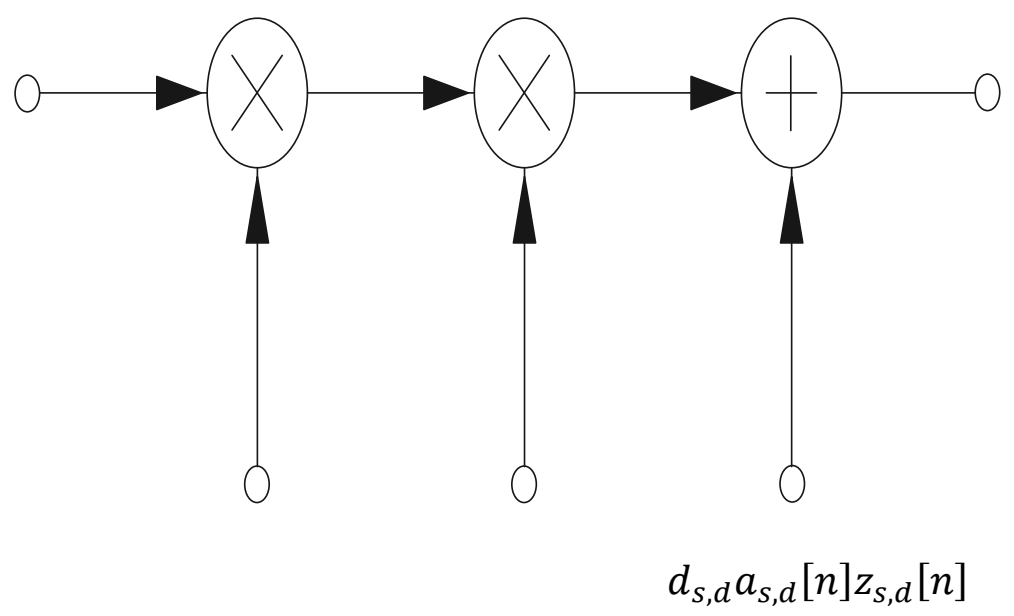

Figure. 6: Channel model 
Noise: The main sources of noise in an underground wireless network are interference and electrical components such as amplifiers. The scalar $\mathrm{z}_{\mathrm{s}, \mathrm{d}}[\mathrm{n}]$ can therefore be replicated as the addition of two Gaussian distributed, locally isolated, and zero mean with variation $\sigma_{n}^{2}$., noise vectors. $N_{0}=2 \sigma_{n}^{2}$ will be the overall noise intensity [28].

\subsubsection{Signal to Noise Ratio}

The signal-to-noise ratio (SNR) is a commonly used metric for determining signal strength at a given location.

$$
\operatorname{SNR}=\left(\frac{S}{N_{O}}\right)=\frac{\left|h_{s, r}\right|^{2} \varepsilon}{N_{O}}
$$

In (3.2) $\xi=E\left[\left|x_{s}\right|^{2}\right]$ indicates the transmitted signal's energies as well as the noise's overall power.

\subsubsection{Path Loss and Fading}

The impacts of fading and free-space path loss, both of which are included in $h_{s, d}=p l_{s, d} a_{s, d}$

Makes a particular in the entire process might drastically alter the channel's characteristics and, as a result, the signal strength [27]. Fading is a signal-changing effect that involves attenuating the information and applying a phase shift to it. A zero mean, complex Gaussian random variable with $a_{s, d}$. Variances can be used to represent the fading coefficient $a_{s, d}{ }^{2}$. This means that the angle $a_{s, d}$ is Rayleigh distributed and that the magnitude $? a_{s, d}$ is uniformly distributed on the range $[0,2]$.

\subsubsection{Block Fading}

In a rapid fading channel, the channel characteristic changes within one explosion of information.This impact is taken into account by the block fading channel model. The explosion has been split down into manageable pieces, or frames, which might possibly be considered be able to tune in on a regular basis characteristics. The block size must be adequate to permit for accurate estimation of the channel characteristic [13] . The receiver knows the amplitude and angle of the block's fading coefficient a $(\mathrm{s}, \mathrm{d})$. There's a good 
chance that burst mistakes will occur in a block fading channel, which means that there will be a lot of errors in one block. With an error-correcting code, such bursts of faults are extremely hard to correct [26]. To avoid them, the signal can be interleaved to disperse the mistakes consistently across the entire signal, as seen in Figure 7. Although the interleaving and coding block are not replicated, they are presumed to exist. As a result, while simulating such a transmission, it makes no difference how the faults are dispersed across the entire signal [14]. The average bit error ratio is the only thing that matters (BER). The signal should be transported over as many separate channel properties as feasible to obtain an accurate result.

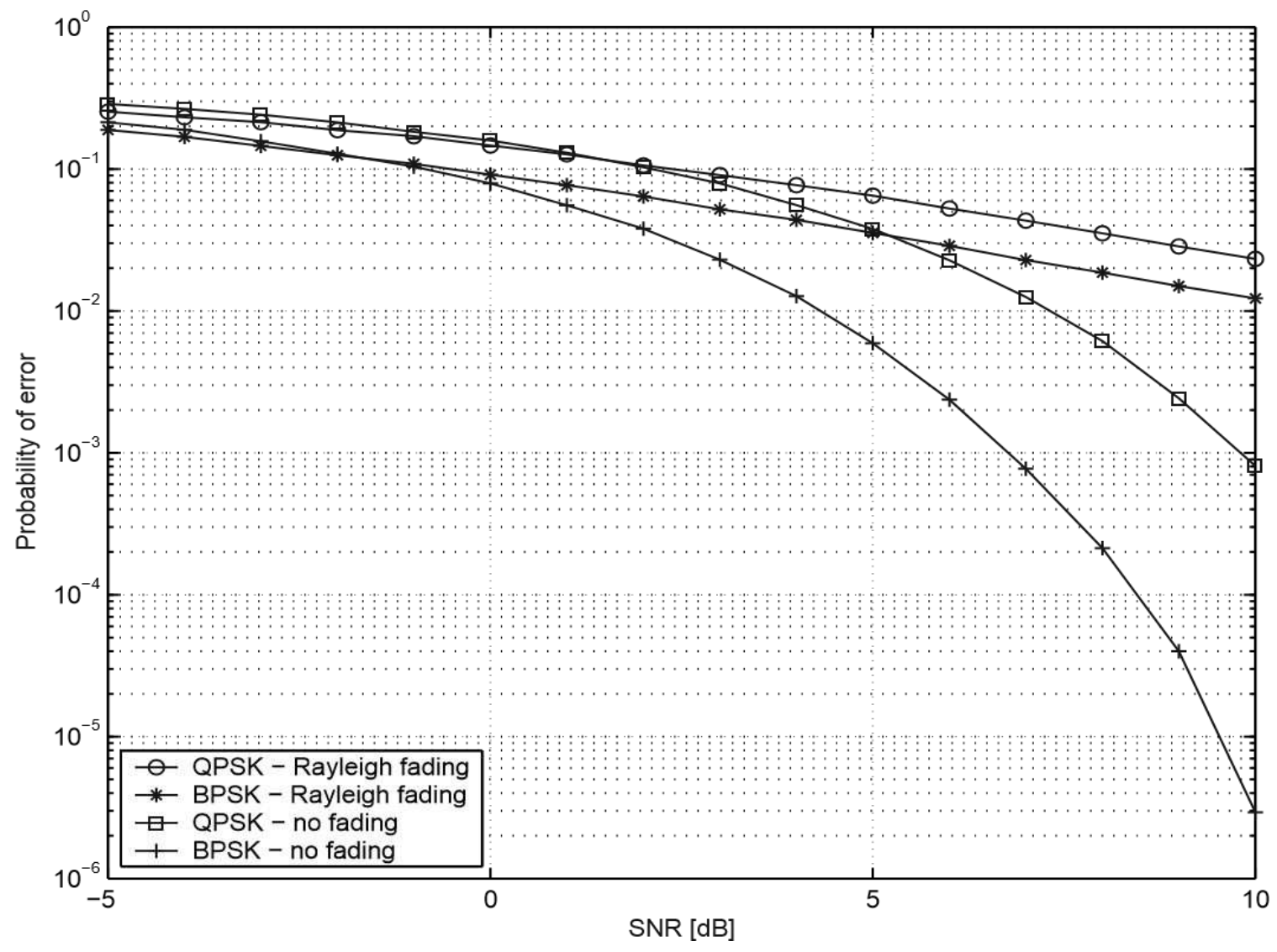

Figure 7: Compared to the non-faded channel, a Rayleigh faded channel has a much 3.4 Receiver Model stronger effect.

Symbol by symbol, the receiver detects the received data. The symbol/bit is recognized as in the case of a BPSK modulated signal.

$$
\hat{\mathrm{y}}_{d}[n]=\left\{\begin{array}{l}
+1\left(R_{e}\left\{y_{d}[n]\right\} \geq 0\right) \\
-1\left(R_{e}\left\{y_{d}[n]\right\}<0\right)
\end{array}\right.
$$


There are two bits sent each symbol in a QPSK modulated signal, which are recognized

$$
\operatorname{asy}_{d}[n]=\left\{\begin{array}{c}
{[+1,+1]\left(0^{\circ} \leq \nabla y_{d}[n]<90^{\circ}\right)} \\
{[-1,+1]\left(90^{\circ} \leq \nabla y_{d}[n]<180^{\circ}\right)} \\
{[+1,+1]\left(-90^{\circ} \leq \nabla y_{d}[n]<0^{\circ}\right)} \\
{[+1,+1]\left(-180^{\circ} \leq \forall y_{d}[n]<-90^{\circ}\right)}
\end{array}\right.
$$

\subsection{BER of a Single Link Transmission}

The signals strength perceived at the destinations is determined by the channel's SNR and modulation method. Table 1 summarizes the theoretical probability of a bit error.

Table 1: For a single link transmission, this is the theoretical BER. The average signal-to-noise ratio is denoted by b. $\gamma_{b}=\frac{\varepsilon}{2 \sigma^{2}} E\left(a^{2}\right)$, where $E\left(a^{2}\right)=a^{2}$.

\begin{tabular}{|l|l|c|}
\hline Modulation Type & No Fading $\mu \mathrm{q}$ & Rayleigh Fading \\
\hline BPSK & $P b=Q 2 \sigma \varepsilon^{2}$ & $P_{b}=\frac{1}{2}\left(1-\sqrt{\frac{\varepsilon_{b}}{1+\gamma_{b}}}\right)$ \\
\hline QPSK & $P b=Q 2 \sigma \varepsilon^{2}$ & $P_{b}=\frac{1}{2}\left(1-\sqrt{\frac{\varepsilon_{b}}{2+\gamma_{b}}}\right)$ \\
\hline
\end{tabular}

This experiment, which is seen in Figure 7, shows how fading has a detrimental impact on signal strength. The figure also demonstrates that the BPSK modulated signal performs 3dB better than the QPSK modulated signal in average.

\section{EXISTING PROBLEM IN COOPERATIVE DIVERSITY}

Two well-known cooperative communication strategies are amplify and forward (AF) and decode and forward (DF) [25]. A new error analysis approach has been proposed for single-relay cooperative diversity using selection combining. Scaling selection combining was used to evaluate the performance of a single-relay cooperative diversity.

\subsection{Conventional Selection Combining With Multiple Relays}

With numerous relays, the error performance (SEP) is computed using Conventional Selection Combining.

\subsubsection{System model}

A source, numerous relays, and a destination are all part of the system model under examination. Multiple relays are used to transmit data from the source to the destination. 
All of the channels are considered to be independent and identically distributed and to fade flatly[24] . The procedure is divided into two sections. The source transmits the BPSK data symbol to both the destination and the relays in phase 1 . In phase 1 , the complicated baseband received signal at the destination and relays is represented as

$$
\begin{aligned}
& r_{s d}=h_{s d} s+n_{s d}, \\
& r_{s r_{i}}=h_{s r_{i}} s+n_{s r_{i}}, \quad i=1 \ldots . . N,
\end{aligned}
$$

where $h_{s d}$ and $h_{s r_{i}}$ are the source-to-destination and source-to-ith-relay random complex fading gains, accordingly, $\mathrm{s}$ is the transmitted BPSK symbol of the source with energy $2 E_{S}\left(s \in\left\{-\sqrt{E_{s}}, \sqrt{E_{s}}\right\}\right)$, and $n_{s d}$ and $n_{s r_{i}}$ are the additive noises of the source-todestination and source-to-i ${ }^{\text {th }}$ relay connection. $h_{s d}$ and $h_{s r_{i}}$ are circular zero-mean complexes that are independent Random variables with Gaussian variances $\Omega_{s d}$ and $\Omega_{s r}$, correspondingly, and are not affected by additive noise due to independent Rayleigh fading. The additive noises $n_{s d}$ and $n_{s r_{i}}$ are complicated with zero mean circular Gaussian random variables with variance $2 N_{0}$ and a $C \mathcal{N}\left(0,2 N_{0}\right)$ distribution, respectively [15] . The relay detects the transmitted BPSK symbol in phase 2 and obtains the detected symbol s. The detected symbol is then forwarded to the destination by the relays. Phase 2 models the complicated baseband received signal at the destination as

$$
r_{r d_{i}}=h_{r d_{i}} \hat{s}+n_{r d_{i}}, \quad i=1 . . N
$$

While $h_{r d}$ and $n_{r d_{i}}$ are the ith-relay-to-destination channel's fading gain and additive noise, respectively, and are represented as $\operatorname{C~} C \mathcal{N}\left(0, \Omega_{r d}\right)$ and $\left(0,2 N_{0}\right)$, respectively. It's worth noting that $h_{r d_{i}}$ and $n_{r d_{i}}$ are unrelated to $h_{s d}, h_{s r_{i}}, n_{s d}, n_{s r_{i}}$ and one another. The destination, we assume, is aware of all of the channels' diminishing gains. The source-todestination, source-to-relay, and relay-to-destination channels' instantaneous SNRs are denoted as, respectively [16].

$$
\begin{aligned}
\gamma_{s d} & =\frac{E_{s}}{N_{0}}\left|h_{s d}\right|^{2}, \gamma_{s r_{i}}=\frac{E_{s}}{N_{0}}\left|h_{s r_{i}}\right|^{2}, i=1 \ldots L, \\
\gamma_{r d_{i}} & =\frac{E_{s}}{N_{0}}\left|h_{r d_{i}}\right|^{2}, i=1 \ldots L,
\end{aligned}
$$


The mean SNRs are calculated as follows:

$$
\begin{aligned}
\Gamma_{s d}=\mathrm{E}\left[\gamma_{s d}\right]=\frac{E_{s} \Omega_{s d}}{N_{0}}, \Gamma_{s r} & =E\left[\gamma_{s r_{i}}\right]=\frac{E_{s} \Omega_{s r}}{N_{0}}, \\
\Gamma_{r d} & =E\left[\gamma_{r d_{i}}\right]=\frac{E_{s} \Omega_{r d}}{N_{0}},
\end{aligned}
$$

\subsubsection{Conventional Decision Rule}

Let us consider $L$ number of relays,

$$
\begin{gathered}
\text { if } \gamma_{s d}>\gamma_{r d_{i}}, \quad i=1 \ldots L \\
\text { then } \\
\text { select } S-D \text { link } \\
\text { elseif } r_{s d}<\gamma_{r d_{i}}, \quad i=1 \ldots L
\end{gathered}
$$

select ith - source - to - relay destination link

\subsubsection{Algorithm}

This algorithm investigates the error performance of cooperative diversity networks using BPSK modulation.

\section{Require:}

$$
\begin{aligned}
& h_{a b_{i, N}} \sim \mathrm{C} \mathcal{N}(0,1) \text { for } a b \in\{s d, s r, r d\}, \\
& 1 \leq i \leq N_{b}, N=1 \ldots L, n_{a b_{i, N}} \sim C \mathcal{N}\left(0,2 N_{0}\right)
\end{aligned}
$$

$N_{b}$-total number of bits

$$
\text { Where, } L \text { - total number of relays }
$$

Ensure: $S, \hat{s}_{N}, \hat{s}_{s d}$ and $\hat{s} \in \grave{A}=\left\{\sqrt{2 E_{s}},-\sqrt{2 E_{s}}\right\}$

for $N=1 \ldots L$

$S E=0$

$$
\text { while } i=1 \ldots N_{b}
$$

\section{Phase 1:}

$$
\begin{aligned}
& r_{s r_{i, N}}=h_{s r_{i, N}} s_{i}+n_{s r_{i, N}} \\
& r_{s d_{i}}=h_{s d_{i}} s_{i}+n_{s d_{i}}
\end{aligned}
$$

\section{Source-to-relay link detection at relay}

$\hat{S}_{i, N}=\arg \left\{\max _{s \in \grave{A}} \operatorname{Re}\left(s_{i} * h_{s r_{i, N}} * h_{s r_{i, N}}\right)\right.$

\section{Source-to-destination detection at destination}

$\hat{S}_{i, s d}=\arg \left\{\max _{s \in \grave{A}} \operatorname{Re}\left(s_{i} * h_{s d_{i}} * h_{s r_{i}}\right)\right.$

Phase 2:

$r_{r d_{i, N}}=h_{r d_{i, N}} \hat{s}_{i, N}+n_{r d_{i, N}}$ 


\section{Modified Selection Combining:}

assign $\gamma_{s d}=\frac{E_{s}}{N_{0}}\left|h_{s d_{i}}\right|^{2}$ and $\gamma_{r d}=\frac{E_{s}}{N_{0}}\left|h_{r d_{i}}\right|^{2}$

Conventional Decision rule:

$$
\begin{aligned}
& \hat{\hat{S}}_{i}=\arg \left\{\max _{s \in A}, \operatorname{Re}\left(s_{i} * h_{r d_{i, N}} * \gamma_{r d_{i, N}}\right)\right\} \\
& \text { else } \\
& \hat{\hat{S}}_{i}=\arg \left\{\max _{s \in A}, \operatorname{Re}\left(s_{i} * h_{s d_{N}} * \gamma_{s d_{i}}\right)\right\} \\
& \text { end if } \\
& \text { SEP calculation: }
\end{aligned}
$$

$$
\begin{gathered}
\text { if } \hat{\hat{S}}_{i} \neq S_{i} \text { then } \\
S E=S E+1 \\
\text { end if } \\
\text { end while } \\
S E P=S E / N_{b}
\end{gathered}
$$

Proposed Selection combining with multiple relays: Error performance (SEP) is calculated using Proposed Selection Combining with multiple relays.

\subsubsection{Representation of the system}

Consider a cooperative diversity structure with a source, a relay, and a destination. Both the destination and the relay received an MPSK symbol from the source [31]. The relay retransmits the MPSK symbol to the destination using the DF protocol [21]. There is symbol-by-symbol transmission available. Each of the SD, SR, and RD links is selfcontained and fades in a flat Rayleigh pattern. The complex MPSK symbol is part of the complex-constellation and has 2Es of energy [18] .

$\mathrm{S}=\left\{S_{1}, S_{2}, \ldots, S_{M}\right\}$, as defined by

$$
S_{m}=\sqrt{2 E_{s}} \exp \left(\frac{j 2 \pi(m-1)}{M}\right), \quad m=1, \ldots, M
$$

where $j=\sqrt{-1}$. The symbol transmission is split into two orthogonal time frames. The source broadcasted the MPSK symbols to both in the first instance, the destination and the 
relay frame. In time slot 1 , the baseband complex receiving signal at the destination and at the relay is represented as

$$
\begin{aligned}
& r_{s d}=h_{s d} s+n_{s d}, \\
& r_{s r_{i}}=h_{s r_{i}} s+n_{s r_{i}}, \quad i=1 \ldots N
\end{aligned}
$$

respectively, where $h_{s d}$ and $h_{r d}$ are the SD and SR links' random complex fading gains, accordingly. Furthermore, $\mathrm{n}_{\mathrm{sd}}$ and $\mathrm{n}_{\mathrm{rd}}$ are the SD and SR links' additive white Gaussian noises, correspondingly [22]. The fading gains $h_{s d}$ and $h_{r d}$ are represented by $s_{d}$ and $s_{r}$, respectively, as circularly symmetric complex Gaussian random variables with zero mean and variance [33]. The noises are represented as $\mathrm{CN}\left(0,2 \mathrm{~N}_{0}\right)$, a variance $2 \mathrm{~N}_{0}$ zero mean complex Gaussian random variable. In the allotted time window, the relay sends the indicator $\mathrm{S}$ to the destination. The transmitted complex baseband signal is given by at the destination [17].

$$
r_{r d}=h_{r d} \hat{S}+n_{r d}
$$

The source-to-destination, source-to-relay, and relay-to-destination instantaneous SNRs channels are denoted as, accordingly.

$$
\begin{gathered}
\gamma_{s d}=\frac{E_{s}}{N_{0}}\left|h_{s d}\right|^{2}, \gamma_{s r_{i}}=\frac{E_{s}}{N_{0}}\left|h_{s r_{i}}\right|^{2}, i=1 \ldots L, \\
\gamma_{r d_{i}}=\frac{E_{s}}{N_{0}}\left|h_{r d_{i}}\right|^{2}, i=1 \ldots L,
\end{gathered}
$$

The respective mean SNRs are calculated as follows:

$$
\begin{aligned}
\Gamma_{s d}=\mathrm{E}\left[\gamma_{s d}\right]=\frac{E_{s} \Omega_{s d}}{N_{0}}, \Gamma_{s r}=E\left[\gamma_{s r_{i}}\right]=\frac{E_{s} \Omega_{s r}}{N_{0}}, \\
\Gamma_{r d}=E\left[\gamma_{r d_{i}}\right]=\frac{E_{s} \Omega_{r d}}{N_{0}},
\end{aligned}
$$

where $h_{r d}$ and $n_{r d}$ denote the RD link's complicated fading gain and additive white Gaussian noise[32]. We assume that the destinations have channel state information (CSI) for all connections and that the relay has CSI for the SR link. [19] The decision rule obtains the final recognized symbol S derived from the provided scheme at the destination, which is denoted as the instant SNRs of the SD, SR, and RD links, respectively [23] . 


\subsubsection{Proposed Decision Rule}

Let us consider $L$ number of relays,

\subsubsection{Algorithm}

$$
\begin{gathered}
\text { if } \gamma_{s d}>\min \left(\gamma_{s r_{i}}, \gamma_{r d_{i}}\right), \quad i=1 \ldots L \\
\text { then } \\
\text { select } S-D \text { link } \\
\text { elseif } \gamma_{s d}<\min \left(\gamma_{s r_{i}}, \gamma_{r d_{i}}\right), \quad i=1 \ldots L \\
\text { select ith }- \text { source }- \text { to }- \text { relay destination link }
\end{gathered}
$$

BPSK modulation is used in this approach to examine the error performance of cooperative diversity networks.

\section{Require:}

$$
\begin{gathered}
h_{a b_{i, N}} \sim \mathrm{CN}(0,1) \text { for } a b \in\{s d, s r, r d\}, \\
1 \leq i \leq N_{b}, N=1 \ldots L, n_{a b_{i, N}} \sim C \mathcal{N}\left(0,2 N_{0}\right) \\
\text { Where, } L \quad \text { - total number of relays } \\
N_{b} \text { - total number of bits }
\end{gathered}
$$

Ensure: $S, \hat{\mathrm{s}}_{N}, \hat{\mathrm{s}}_{s d}$ and $\hat{s} \in \grave{A}=\left\{\sqrt{2 E_{s}},-\sqrt{2 E_{s}}\right\}$

$$
\begin{gathered}
\text { for } N=1 \ldots L \\
\text { while } i=1 \ldots N_{b}
\end{gathered}
$$

Phase 1:

$$
\begin{aligned}
& r_{s r_{i, N}}=h_{s r_{i, N}} s_{i}+n_{s r_{i, N}} \\
& r_{s d_{i}}=h_{s d_{i}} s_{i}+n_{s d_{i}}
\end{aligned}
$$

Source-to-relay link detection at relay

$$
\hat{S}_{i, N}=\arg \left\{\max _{s \in \grave{A}} \operatorname{Re}\left(s_{i} * h_{s r_{i, N}} * h_{s r_{i, N}}\right)\right.
$$

\section{Source-to-destination detection at destination}

$$
\hat{S}_{i, s d}=\arg \left\{\max _{s \in \grave{A}} \operatorname{Re}\left(s_{i} * h_{s d_{i}} * h_{s r_{i}}\right)\right.
$$

Phase 2:

$$
r_{r d_{i, N}}=h_{r d_{i, N}} \hat{\mathrm{s}}_{i, N}+n_{r d_{i, N}}
$$

\section{Modified Selection Combining:}

assign $\gamma_{s d}=\frac{E_{s}}{N_{0}}\left|h_{s d_{i}}\right|^{2}$ and $\gamma_{r d}=\frac{E_{s}}{N_{0}}\left|h_{r d_{i}}\right|^{2}$

Decision rule:

$$
\begin{gathered}
\text { if } r_{s d_{N}}<\min \left(r_{s r_{N}}, r_{r d_{N}}\right) \\
\hat{S}_{i}=\arg \left\{\max _{s \in A}, \operatorname{Re}\left(s_{i} * h_{r d_{i, N}} * r_{r d_{i, N}}\right)\right\} \\
\text { else }
\end{gathered}
$$


$\hat{\hat{S}}_{i}=\arg \left\{\max _{s \in A}, \operatorname{Re}\left(s_{i} * h_{s d_{i, N}} * r_{s d_{i, N}}\right)\right\}$

end if

SEP calculation:

$$
\text { if } \hat{S}_{i} \neq S_{i} \text { then }
$$

$S E=S E+1$

end if

end while

$S E P=S E / N_{b}$

\section{EXPERIMENTAL RESULS AND OUTPUTS}

\subsection{Results of conventional Selection combining with multiple relays}

Figure.8 shows the Symbol Error Probability of the MPSK modulation over a Additive white Gaussian noise channel (AWGN) without the effect of fading.

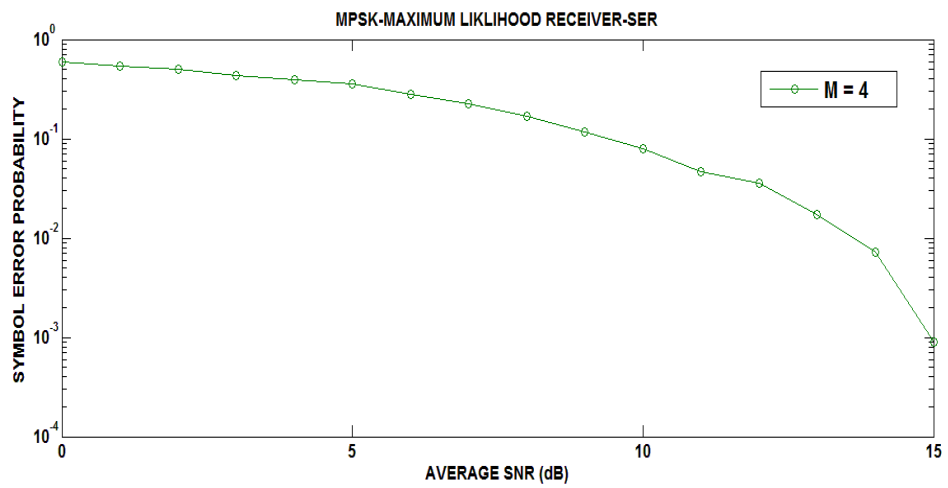

Figure.8 : SEP of QPSK (M=4) using ML detection

It coveys that the experimental results matches with the theoretical value of SEP of the QPSK (M = 4, MPSK) which is found using the Q-function. It proves that the SEP reduces with the increasing value of Signal-to-Noise ratio (SNR) value. As the signal power increases the probability of error in the detection of symbol at receiver decreases.Figure.9 compares the error performance of different modulation techniques of $\operatorname{MPSK}(M=2,4,8)$. It evident from the below plot that the SEP reduces with increasing order of modulation. 


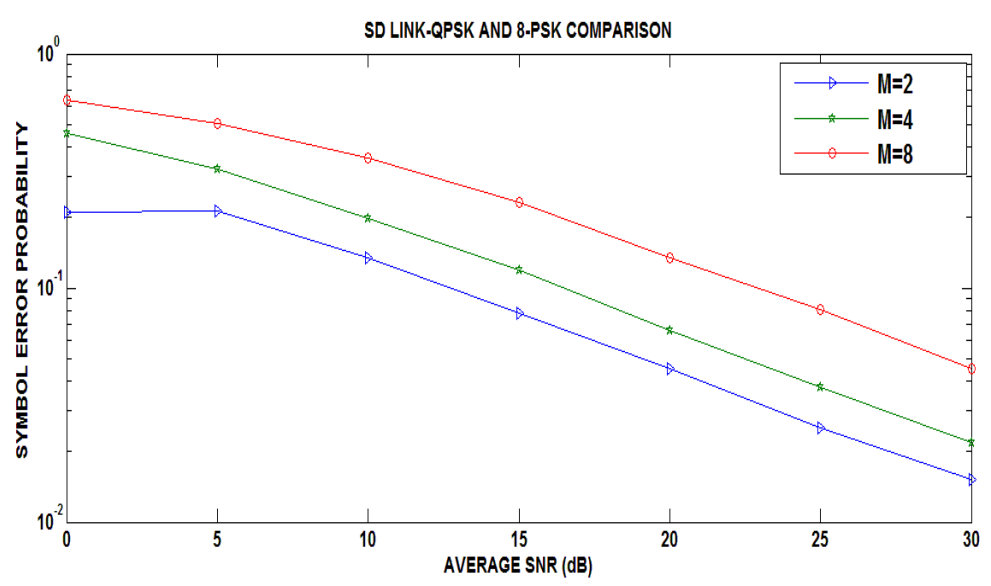

Figure.9 :Comparison of modulations $(M=2,4,8)$ under direct link

Figure.10 compares the error performance of the non-cooperation and cooperation networks.

It is evident that SEP of cooperative network with single relay outperforms the noncooperation network by $5 \mathrm{~dB}$ at $10^{-2}$ SEP. Figure.11 gives the performance improvement shown by the cooperative network by the introduction of different values of alpha $(0.01,0.1$, 1 , and 10) which is proportional to the source to relay distance. The findings demonstrate that the distance between the source and the relay decreases the SEP decreases. As the source to relay distance increase its proportionate alpha value decreases. Thus more the alpha value less is the value of SEP (order of $10^{-3}$ ).

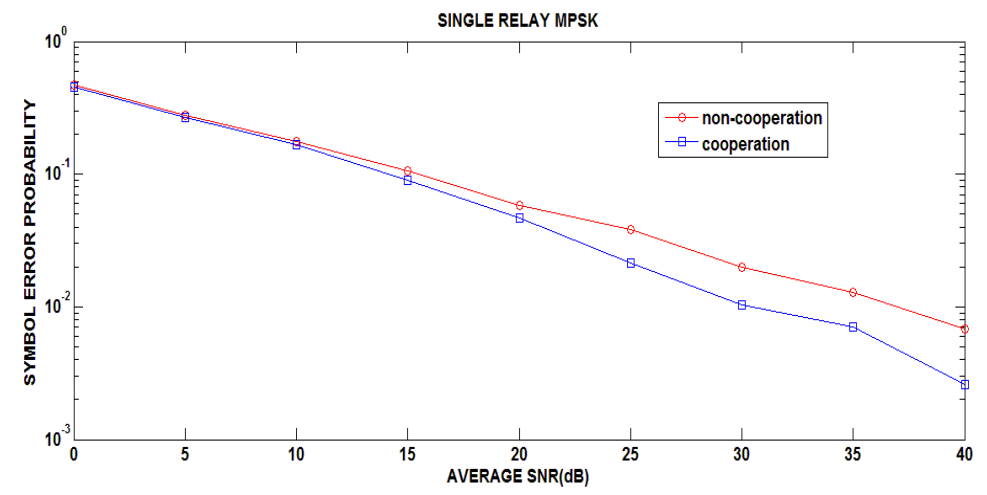

Figure.10: SEP of single relay MPSK $(M=4)$

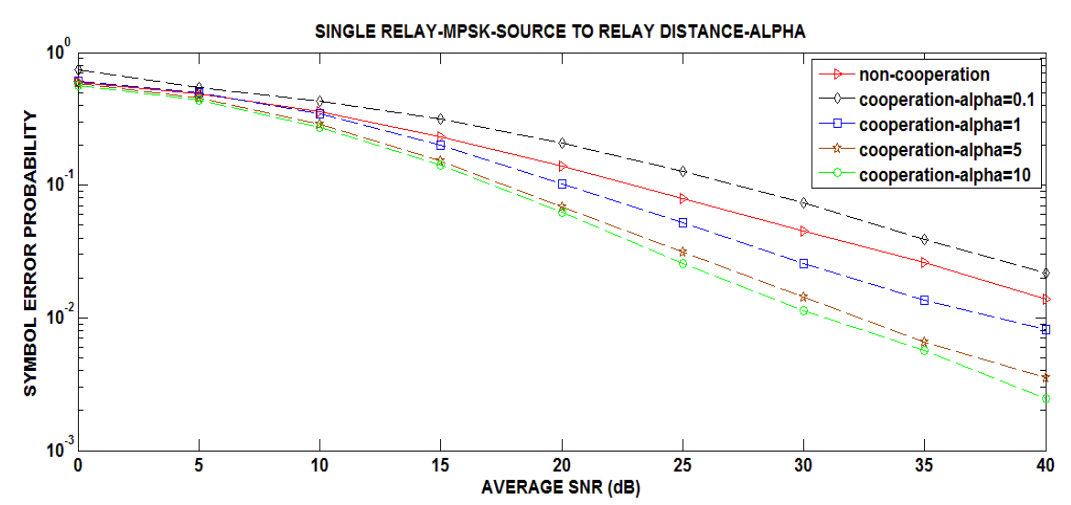


Figure.11: SEP of single relay MPSK $(M=4)$ with source to relay information

Figure.12 shows the plot of Pe versus SNR $(\mathrm{dB})$ in a cooperative network with varying numbers of relays, $\mathrm{N}\left(\right.$ with $\left.\Gamma_{s d}=\Gamma_{s r}=\Gamma_{r d}\right)[20]$. We discovered that as the SNR and $\mathrm{N}$ (number of relays) increase, the SEP drops. However, with higher values of $\mathrm{N}$, it tends to overload, indicating that the cooperative network is performing at its best.

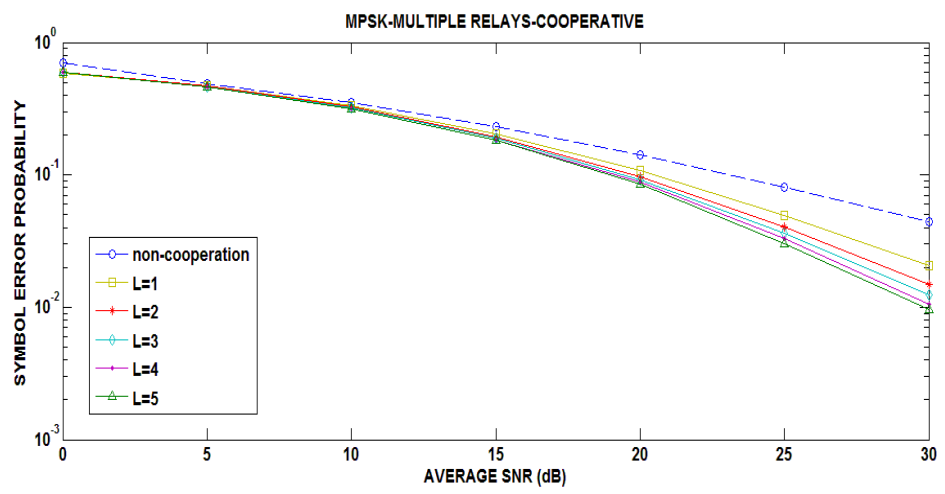

Figure. 12: SEP of MPSK multiple relays

\subsection{Results of Proposed Selection combining with multiple relays}

Figure 13 shows the diagram of the SEP $P_{e}$ vs the mean SNR of the transmitter to receiver channel_sd (with sd=_sr= rd) for various Values of N. We showed that when the mean SNR and $\mathrm{N}$ rise, the error performance also improves; nevertheless, bigger

$\mathrm{nN}$ values tend to attain saturation.

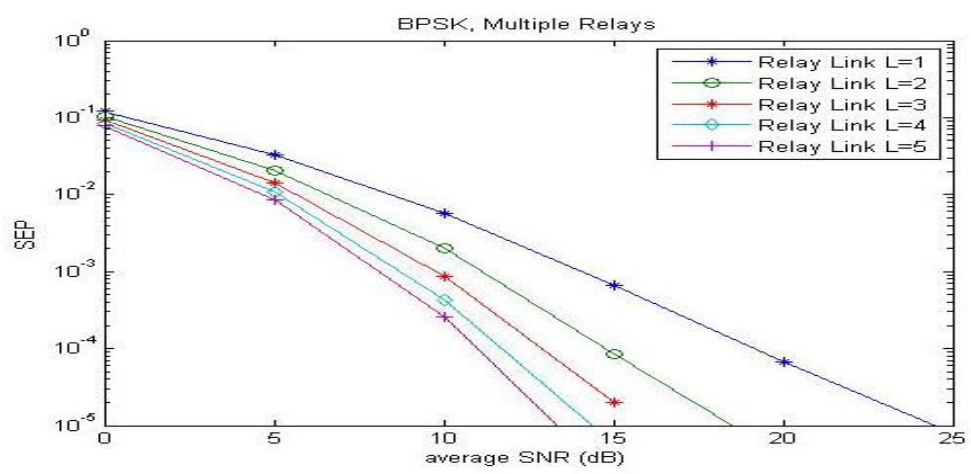

Figure.13: Performance analysis for multiple relays

We now evaluated the proposed technique to a traditional selection combining technique, in which the route with the highest immediate SNR is chosen and the constraint is given by

$$
\hat{\mathrm{s}}=\left\{\begin{array}{l}
\sqrt{2 E_{s}} \operatorname{sgn}\left(\operatorname{Re}\left(h_{s d}^{*} r_{s d}\right)\right) \text { if } r_{s d}>r_{r d}, \\
\sqrt{2 E_{s}} \operatorname{sgn}\left(\operatorname{Re}\left(h_{r d}^{*} r_{r d}\right)\right) \text { if } r_{s d}>r_{r d},
\end{array}\right.
$$


Figure 14 shows that as the mean SNR rises, so does the error probability, and as the modulation sequence rises, so does the error performance.

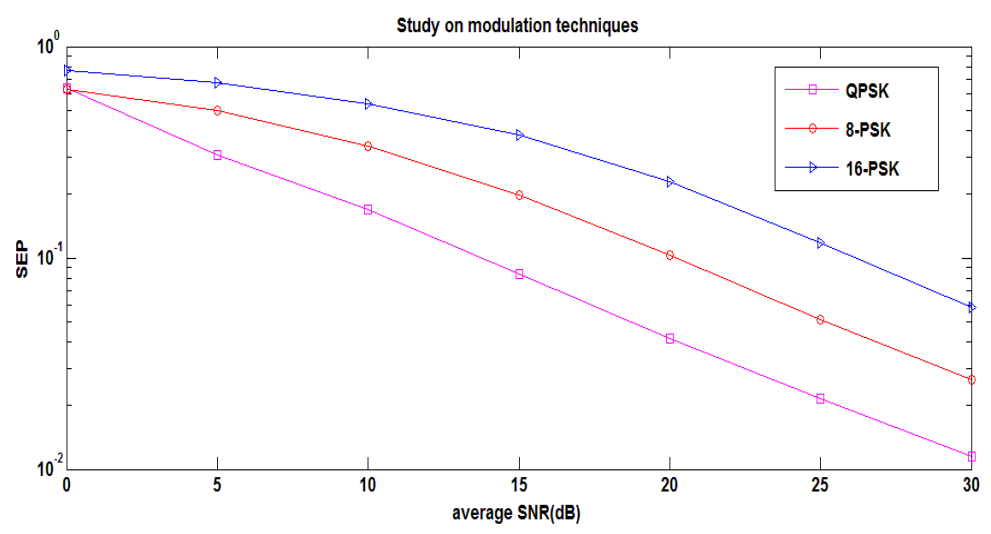

Figure.14: SEP versus Mean SNR for various modulation orders $(M=4,8,16)$.

The SEP vs. the SNR mean for varying alpha value is shown in Figure. 15. The alpha is ratio of the average SNR $\left(\Gamma=\Gamma_{s d}=\Gamma_{r d}\right)$ to the mean SNR of the connection from the sources to the relay $\left(\Gamma_{s r}\right)$. The values of the alpha are[0.01,0.1,1,10]. We notice this as the alpha value raises the error performance enhanceswhich indicates when the source and relay nodes are closer in space then the performance is better. Plots of $P_{\mathrm{e}}$ for proposed scheme and conventional selection combining when $\Gamma_{s d}=\Gamma_{s r}=\Gamma_{r d}$ are shown in Figure.16. We observe that the suggested technique outperforms traditional selection combining in terms of SNR gain by a significant margin. At a SEP of 103, for example, an SNR gain of around $7 \mathrm{~dB}$ over standard selection combining and $11 \mathrm{~dB}$ over noncooperation is realized. The results indicate that collaboration provides significant benefits, and the proposed technique outperforms its conventional counterpart.

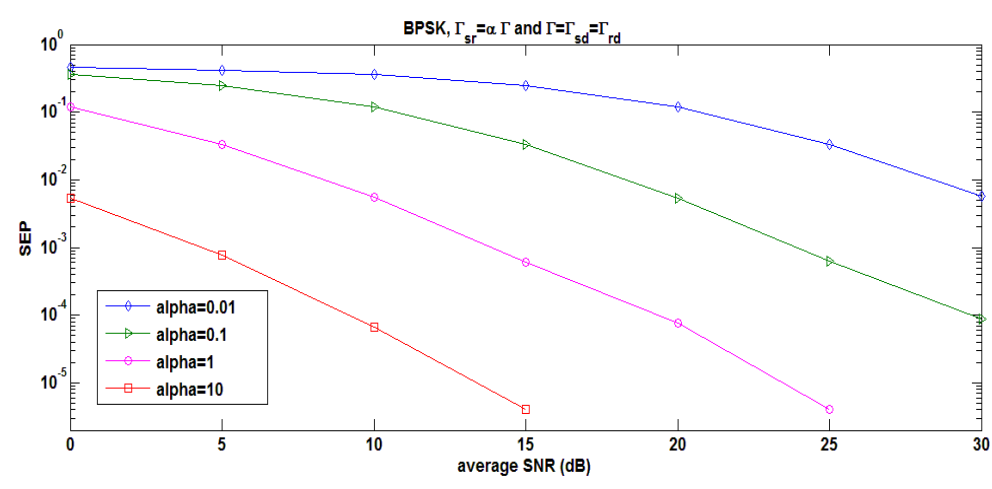

Figure. 15: Investigating SEP for various values of source to relay average SNRs. 


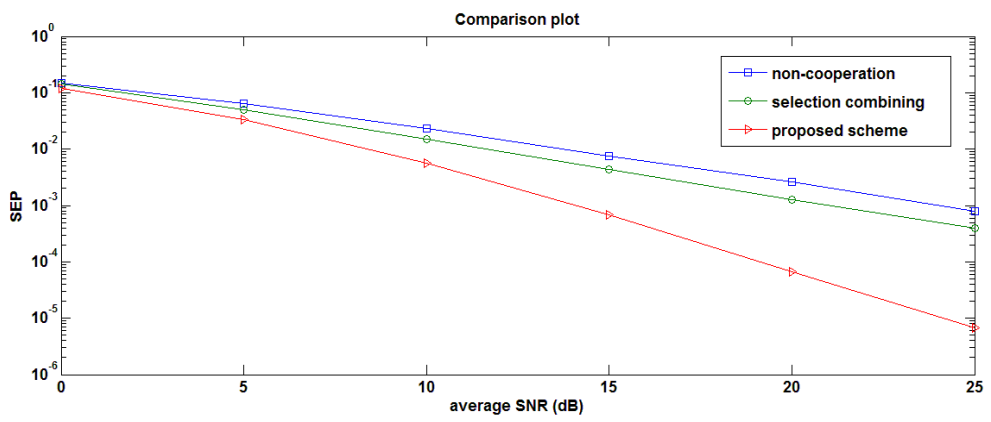

Figure. 16: Comparison with other competing schemes.

\section{Comparative Analysis}

From the Figure.17., The proposed selection combining scheme shows a comparative improvement of $25 \mathrm{~dB}$ at $10^{-2} \mathrm{SEP}$. The conventional selection combining gets a maximum of $10^{-2}$ at $30 \mathrm{~dB}$ SNR. Here the source to relay information is omitted and the fading channel of Rayleigh fading channel with AWGN channel is considered.

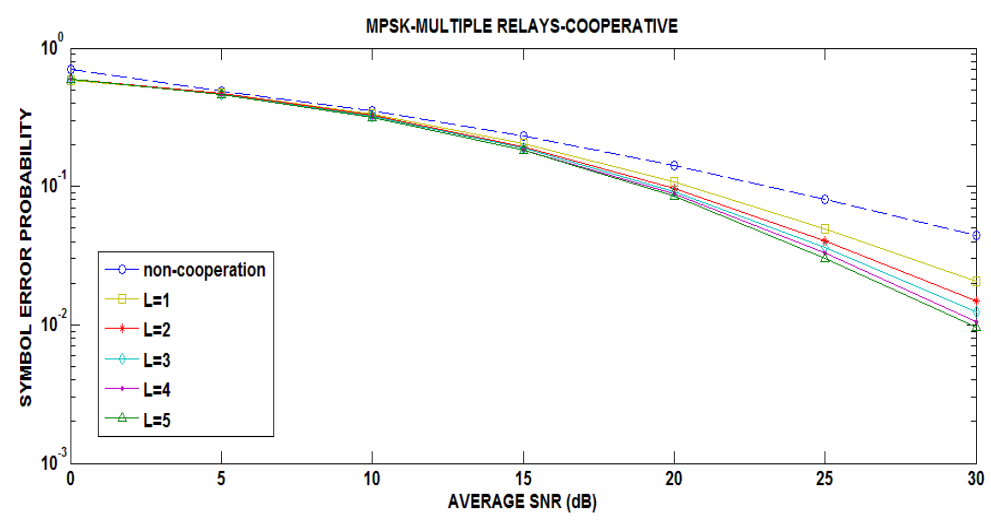

Figure.17: SEP of MPSK multiple relays using conventional selection combining.

SEP has improved immensely when compared to the usual selection combining strategy, as shown in Figure.18, which represents the graph of Pe vs the SNR in $\mathrm{dB}$. On the $30 \mathrm{~dB}$ scale, there is a 10-5 enhancement. This is a 10-2 enhancement, which is particularly 
important in terms of SEP, as it provides communications more consistent and of high

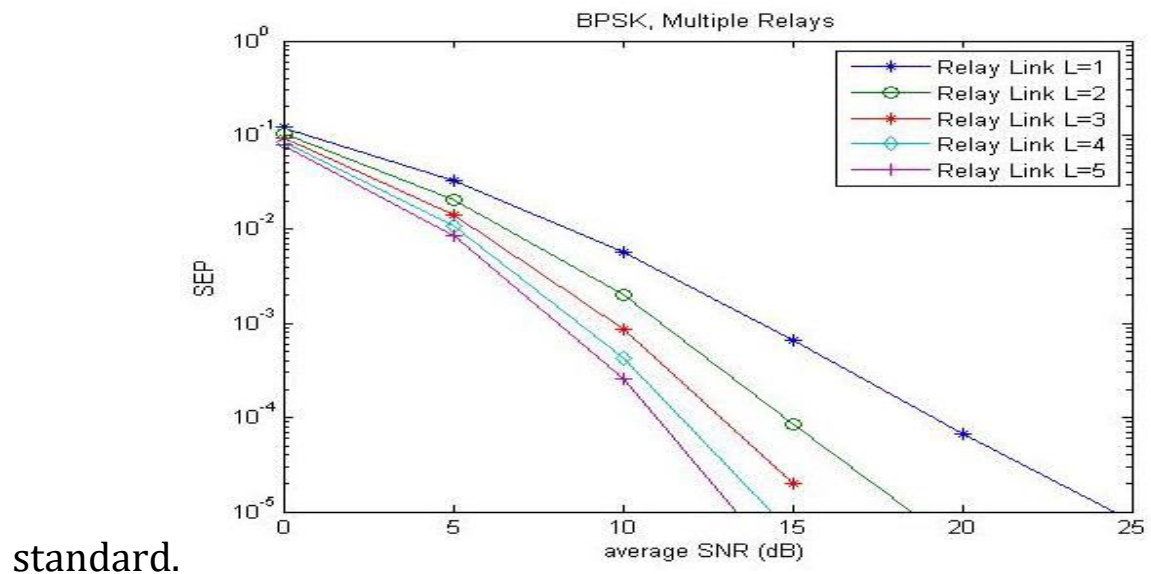

Figure.18: Performance analysis for multiple relays using proposed scheme

Table 2 compares the SEP of the suggested system's selection combining rule to that of the conventional system. The comparison is made for different values of $\mathrm{N}$ (Number of relays).

Table.2: Comparison SEP of the conventional and proposed scheme

\begin{tabular}{|l|l|l|}
\hline SNR(dB) & $\begin{array}{l}\text { SEP } \\
\text { (Conventional scheme) }\end{array}$ & $\begin{array}{l}\text { SEP } \\
\text { (Proposed scheme) }\end{array}$ \\
\hline 0 & 0.5943 & 0.0780912 \\
\hline 5 & 0.4608 & 0.0087173 \\
\hline 10 & 0.3136 & 0.0001747 \\
\hline 15 & 0.1813 & 0.0000859 \\
\hline 20 & 0.08496 & 0.0000048 \\
\hline 25 & 0.03042 & 0.0000021 \\
\hline 30 & 0.00957 & 0.0000001 \\
\hline
\end{tabular}

\section{CONCLUSION}

This paper illustrates the potential advantages of applying cooperative diversity to enhance the productivity of an underground wireless transmission. Establishing underground sensor network with a third node acting as a relay achieves diversity. Information is moved straight from the baseline to the cellular telephone or through a relay node. A network like this has been modelled to examine how different diversity policies and combining methods perform. Regardless of the combined mechanism utilized at the reception, the AAF protocols outperformed the DAF protocol. However, it should be noted that no error-correcting coding was appended to the signals that was sent. As a result, it was not able to fully utilize DAF protocol. The enchanted genie was used to mimic an error correcting code in order to obtain a sense of the DAF protocol's capabilities. A systems that used the DAF protocol in conjunction 
with a magic genie performed markedly superior to one that used the AAF protocol. The merging methodology adopted has a significant impact on the error rate at the reception. When AAF is utilized at the relay station, Equal Ratio Combining (ERC), which is easy to accomplish, offers some advantages over single link transmissions. Fixed Ratio Combining (FRC) ought to be employed wherever practicable. This merely involves knowing of the mean channel quality and outperforms the ERC significantly. More advanced combining strategies can be implemented if understanding of the present condition of the channel quality is available. When an approximate estimation of the channel quality is sufficient, the Enhanced Signal-to-Noise Ratio Combining (ESNRC) has demonstrated to be quite effective. The relay's position is critical to its success. When the relay node is at an equivalent or significantly nearer distance from the transmitter and the receiver, the best possible performance is attained. By typically, the relay must be close to the line that connects the two terminals.

Funding Statement: The authors would like to thank King Khalid University for funding this work through Small Research Project under grant number RGP/108/42.

Conflicts of Interest: The authors declare no conflict of interest.

\section{REFERENCES}

1. Isaraf, Z., Ahmed, A., Khan, F.A. (2021) . “Cooperative non-orthogonal multiple access for wireless communication networks by exploiting the EXIT chart analysis",. Journal Wireless Communication Network, 79,. https://doi.org/10.1186/s13638-021-01961-z

2. Nosratinia, Aria \& Hunter, T.E. \& Hedayat, A., (2004) " Cooperative communication in wireless networks. Communications", IEEE Magazine, IEEE. 42. 74 - 80. 10.1109 / MCOM.2004 . 1341264.

3. Guangjun Liang, Qi Zhu , Jianfang Xin, Jiashan Tang, and Tianjiao ZhangPY(2017) ,Performance Analysis of Buffer-Aided Relaying System Based on Data and Energy Coupling Queuing Model for Cooperative Communication Networks", Wireless Communications and Mobile Computing, Volume 2017 ,Article ID 9287489 https://doi.org/10.1155/2017/9287489

4. Kanthavel, R., Dhaya, R.(2022) , Wireless Underground Sensor Networks Channel Using Energy Efficient Clustered Communication. Intelligent Automation \& Soft Computing, vol. 31,no.1, 649-659.

5. Young Gil Kim,Norman C. Beaulieu (2015),Exact BEP of Decode-and-Forward Cooperative Systems With Multiple Relays in Rayleigh Fading Channels, IEEE Transactions On Vehicular Technology, 64,2,823-828 
6. Tian Lu, Peng Liu, Il-Min Kim, Franc,ois Chan, William Read(2015), End-to-End Optimum ML Detection for DF Cooperative Diversity Networks in the Presence of Interference,IEEE Transactions on Underground wireless Communications,14,5,2639 2654.

7. Hyun-Myung Kim, Tae-Kyoung Kim, Moonsik Min,Gi-HongIm (2015), Low-Complexity Detection Scheme for Cooperative MIMO Systems With Decode-and-Forward Relays, IEEE Transactions On Communications,63,1,94 - 106

8. Bumsoo Lee, Chungyong Lee(2014),Performance Analysis of Incremental Hop Selection Scheme With Adaptive Modulation for Cooperative Multi-Hop Networks, IEEE Transactions On Underground wireless Communications,14,1, 435 - 445.

9. Li Fei,Jun Zhang, Qiang Gao, Xiao-Hong Peng (2015),Outageoptimal relay strategy under outdated channel state information in decode-and-forward cooperative communication system, 9,4,441-450

10. Zhengguo Sheng, Jun Fan, Chi Harold Liu, Victor C. M. Leung, Xue Liu, and Kin K. Leung(2015),Energy-Efficient Relay Selection for Cooperative Relaying in Underground wireless Multimedia Networks, IEEE Transactions On Vehicular Technology, 64,3,1156-1170.

11. Xuehua Zhang, Mazen Hasna and Ali Ghrayeb(2016),An Adaptive Transmission Scheme for Two-way Relaying with Asymmetric Data Rates,IEEE Transactions on Vehicular Technology,65,3,1477 - 1491.

12. Qiang Wang, Member, Yue Dong, Xiaodong Xu, Member, and Xiaofeng Tao(2015), Outage Probability of Full-Duplex AF Relaying with Processing Delay and Residual SelfInterference, IEEE Communications Letters,19,5,783 - 786.

13. Tao Li, Pingyi Fan, and Khaled Ben Letaief(2015), Outage Probability of Energy Harvesting Relay-aided Cooperative Networks over Rayleigh Fading Channel, IEEE Transactions on Vehicular Technology,65,2,972 - 978.

14. Qingjiao Song and Walaa Hamouda(2015),Performance Analysis and Optimization of Multi-Selective Scheme for Cooperative Sensing in Fading Channels, IEEE Transactions on Vehicular Technology,65,1,358-366.

15. Yihenew Wondie Marye, Hua-An(2014), Nearest Neighbor Relay Selection with Adaptive odulation for Improved Throughput and Scalability of Cooperative Underground wireless Networks,IEEE International Conference on Intelligent and Advanced Systems (ICIAS).

16. Santhoshkumar, , M. D. Selvaraj(2014),Performance Analysis of Scaled Switching Technique for Cooperative Diversity Systems, IEEE International Conference on Information and Communication Technology.

17. Ahmed EI-Mahdy(2014),Differential Space-Time Coding for Partially-Coherent Decode and Forward Cooperative Communication, IEEEInternational Conference on Engineering and Technology (ICET).

18. S.Muthukumar, K.Balachandar, V.Nagarajan, Kirubakaran.S(2014),Performance Analysis of Threshold based Selective Relaying for Switched Selection Combining, International Conference on Communication and Signal Processing,India.

19. Hamza Umit Sokun,Akram Bin Sediq,Salama Ikki†,Halim Yanikomeroglu(2014), Selective DF Relaying in Multi-Relay Networks With Different Modulation Levels, IEEE ICC Underground wireless Communications Symposium. 
20. Hussain Ali and Maan Kousa(2013) ,Incremental Relaying Protocols for Extended LDPC Coded Cooperative Diversity, Underground wireless Communications and Mobile Computing Conference

21. Marco Di Renzo, Michela Iezzi, and Fabio Graziosi, Member(2013),Error Performance and Diversity Analysis of Multi-Source Multi-Relay Underground wireless Networks with Binary Network Coding and Cooperative MRC, IEEE Transactions on Underground wireless Communications,12,6,2883 - 2903

22. MinChul Ju, Member, Kyu-Sung Hwang, and Hyoung-Kyu Song(2013), Relay Selection of Cooperative Diversity Networks With Interference-Limited Destination, IEEE Transactions on Vehicular Technology,62,9 4658 - 4665

23. Punyawi Jamjareegulgarn(2013),Performance Evaluation for Cooperative DiversityBased Underground wireless Body Area Network",IEEE Communications Letters,18,12,345-351

24. Dhaya, R., Kanthavel, R. \& Ahilan, A (2021) . Developing an energy-efficient ubiquitous agriculture mobile sensor network-based threshold built-in MAC routing protocol (TBMP). Soft Computing, 25, 12333-12342 https://doi.org/10.1007/s00500-02105927-7

25. Tiejun Lv, Zhang Zhang, and Shaoshi Yang(2013),A Low Complexity Approach of Combining Cooperative Diversity and Multiuser Diversity in Multiuser Cooperative Networks, IEEE Transactions On Underground wireless Communications,10,4,6247 6256.

26. Hussain Ali and Maan Kousa(2012),A Novel Approach for using Extended LDPC codes in Cooperative Diversity, 2012 IEEE International Conference on Communication Systems.

27. Qijia Liu, Member, Wei Zhang, Member , Xiaoli Ma, and G. Tong Zhou(2012), Designing Peak Power Constrained Amplify-and-Forward Relay Networks with Cooperative Diversity, International Conference on Communication and Signal Processing.

28. Jun-pyo Hon, Bi Hong, Tae Won Ban and Wan Choi, Member(2012),On the Cooperative Diversity Gain in Underlay Cognitive Radio Systems,IEEE Transactions On Vehicular Technology,64,3,3790 - 3795.

29. Neelesh B. Mehta, Vinod Sharma, Gaurav Bansal(2011),Performance Analysis of a Cooperative System with Rateless Codes and Buffered Relays,IEEE Transactions On Underground wireless Communications,10,4,1069 - 1081.

30. R. Dhaya, P. Indhuja, S. Sinduja and M. Swetha(2015), "Finest power efficient steering Algorithm for Wireless Sensor Networks for surveillance," 2015 IEEE 9th International Conference on Intelligent Systems and Control (ISCO), 1-6, doi: 10.1109/ISC0.2015.7282308.

31. Mukhlif F, Noordin KAB, Abdulghafoor OB, Izam TFTMN (2020) Green communication for cognitive radio networks based on game and utility-pricing theories. PLOS ONE 15(8): e0235953. https://doi.org/10.1371/journal.pone.0235953

32. Haiyang Ding, Jianhua Ge, Daniel Benevides da Costa, Zhuoqin Jiang(2011),A New Efficient Low-Complexity Scheme for Multi-Source Multi-Relay Cooperative Networks, IEEE Transactions On Vehicular Technology, 60,2,716-722.

33. Chan-Ho Choi, Ui-Kun Kwon, Young-Jin Kim, Gi-Hong Im(2010),Spectral Efficient Cooperative Diversity Technique with Multi-Layered Modulation, IEEE Transactions On Communications,58,12,3480 - 3490. 